\title{
Intelligente
}

Automatisierung

im Marketing

\section{Wohin geht die Reise?}

Kaum ein Thema beschäftigt die Marketingdisziplin in den letzten Jahren so sehr wie der erfolgreiche Umgang mit und die Nutzung von Daten. Während im Silicon Valley bereits Marketingprozesse abgezielt wird, welche von artifiziellen Intelligenzen (Al) gesteuert werden, ist die Realität in den meisten Unternehmen noch eher analog. Dieser Artikel formuliert fünf wichtige Empfehlungen, welche Unternehmen in der Definition ihrer eigenen, kundenzentrierten Automatisierungsstrategie beachten sollten: (1) die schrittweise Skalierung von IT-Kompetenzen mit Datengrundlagen, (2) die Grundlagen des Dialogmarketings im Design von automatisierten Prozessen berücksichtigen, (3) den korrekten Einsatz von Automatisierung in der Customer Journey verstehen, (4) die sanfte Integration von Mensch und Maschine vorbereiten und schließlich (5) die Privatsphäre von Kunden respektieren. Zusammen ergeben diese Empfehlungen eine Grundlage, auf der die Reise hin zur intelligenten Automatisierung gebaut und geplant werden kann. 
Kaum ein Thema beschäftigt die Marketingdisziplin in den letzten Jahren so sehr wie der erfolgreiche Umgang mit und die Nutzung von Daten. Was einst mit dem Begriff „Database Management" startete und sich später in den Köpfen der Marketer als „Customer Relationship Management" verankerte, wird heute zusammen mit dem allgegenwärtigen Begriff der artifiziellen Intelligenz (AI) diskutiert. Dabei sprengt der Begriff „AI“ regelmäßig die Vorstellungskraft von Forscher*innen und Anwender ${ }^{\star}$ innen. Die einen beschwören demnächst die ersten „Roboter“ mit der Intelligenz eines Menschen herauf, während andere Use Cases für die Vorhersage von Lieferdaten von Paketen diskutieren. Führt man jedoch die Debatte auf ihren Kern zurück, so gilt es für Unternehmen, Ansatzpunkte und Gestaltungshinweise zu bieten, wie mit hoch entwickelten Automatisierungsansätzen intelligente Lösungen für relevante Herausforderungen in Marketing und Vertrieb entwickelt werden können. Getrieben von (ersten) Ansätzen der artifiziellen Intelligenz sollen komplexe Lernprozesse, analytische Verfahren und Prognosen eine intelligente Automation von Aufgaben und Tätigkeiten realisieren helfen [1].

Damit wird die sogenannte intelligente Automation eine der zentralen Treiber für das Marketing Management in den nächsten Jahren sein. So nennt Gartner die Automatisierung im „Hype Cycle für aufkommende Technologien“ dieses Jahr erneut als wichtigen Megatrend und postuliert, dass diese Entwicklung "unumkehrbar und unausweichlich ist. Alles, was automatisiert werden kann und sollte, wird automatisiert werden " [2].

Vor dem Hintergrund dieser Prognose werfen viele Marketer leicht nervös den Blick auf die Prozesse im eigenen Unternehmen und stellen fest, dass das eigene Automatisierungspotenzial noch nicht ausgeschöpft wurde. Dies ist nicht weiter verwunderlich, besonders in Anbetracht der Anzahl an Digitalisierungsinitiativen, welche aufgrund fehlender strategischer Ausrichtung und Skalierbarkeit von Ressourcen gescheitert sind [3]. Doch es gilt für die Digitalisierung dasselbe wie für die Automatisierung: Es ist weniger ein Ziel, das man erreicht - eher ist es ein langfristiger, iterativer Prozess, der angestoßen werden sollte, wenn man nicht von der Konkurrenz überholt werden will.

Auf dem Weg der Automatisierung stellen sich aus kundenorientierter Sicht verschiedene Herausforderungen. In den letzten Jahren hatten wir die Möglichkeit, verschiedene Projekte im Bereich der Automation zu begleiten. Dabei sind uns (neben sicherlich weiteren wichtigen Aspekten) besonders die in der Folge dargestellten Stolpersteine aufgefallen.

\section{Den „richtigen“ Automatisierungspfad erkennen}

Sowohl in der Praxis [4, 5] als auch in der Forschung [6] wird betont, dass Automatisierung kein einmaliger Effort ist. Jedes Automatisierungsprojekt ist mit dem schrittweisen Aufbau von Datenstrukturen, analytischen Fähigkeiten und spezifischen Marketingkompetenzen verbunden. Dabei ist der Entwicklungspfad in Richtung einer intelligenten Automation mit einigen Vorbedingungen verknüpft, die vorab im Unternehmen zu realisieren sind. Dieser Pfad lässt sich grob in vier Phasen unterteilen, von der jede auf die menschlichen und digitalen Ressourcen der vorherigen aufbaut (siehe Abb. 1):

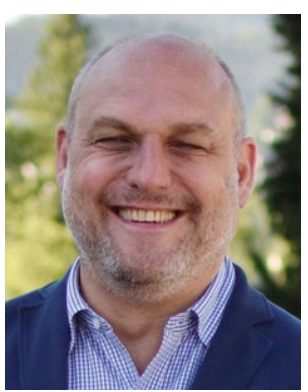

Prof. Dr. Marcus Schögel ${ }^{1}$

ist Professor am Institut für Marketing und Customer Insight an der Universität St. Gallen und Leiter des Masters in Marketing Management. In der Forschung beschäftigt er sich mit den Herausforderungen, die sich aus einer, interaktionsorientierten Marketingperspektive" ergeben. Er arbeitet dabei an der Bewältigung der Herausforderungen von kundenzentrierten Unternehmensstrategien, marktorientierten Veränderungsprozessen in Unternehmen und der Realisierung von erfolgreichen Go-to-market-Strategien marcus.schoegel@unisg.ch

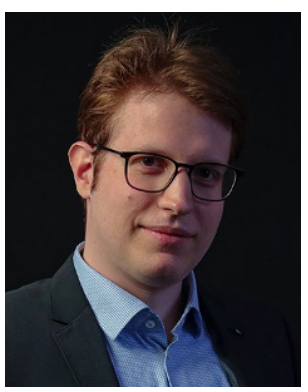

Mauro Luis Gotsch M.A HSG $(\bowtie)$ ist wissenschaftlicher Mitarbeiter am Institut für Marketing und Customer Insight an der Universität St. Gallen. In Forschung und Industriekooperationen beschäftigt er sich schwerpunktmäßig mit strategischem Datenschutzmarketing. Daneben agiert er als Studiengangsleiter des CAS Einkaufsleiter an der Universität St. Gallen. mauro.gotsch@unisg.ch

${ }^{1}$ Institut für Marketing und Customer Insight, University of St. Gallen, St. Gallen, Schweiz 
1. Regelbasiertes Marketing: Viele Unternehmen sind gut vertraut mit dieser Phase, in der einfache Heuristiken und grundlegende Kundendaten (beispielsweise demografische Daten, Einkaufslisten etc.) verwendet werden, um individualisierte Kundeninteraktionen zu gestalten. Oft geschieht dies noch komplett „analog“ und mit unstrukturierten Daten; zum Beispiel durch Verkaufstrainings oder interne Richtlinien. Regelwerke wie „ein Einkauf von Produkten X und Y löst eine Empfehlung für Produkt $\mathrm{Z}$ aus" sind die Grundlage für spätere Automatisierungsprozesse. Einerseits weil ihre Effektivität dank ihrer Einfachheit schnell getestet werden kann, andererseits weil sie wiederum neue Daten generieren, welche für komplexere Heuristiken verwendet werden können.

2. Datengetriebenes Marketing: Der Übergang von Heuristiken zu datengetriebenen Modellen ist fließend - doch sowohl die benötigten Daten als auch das im Unternehmen angesammelte Fachwissen steigen exponentiell. Unstrukturierte Datengrundlagen werden abgelöst durch standardisierte und von Computern besser erfassbare Angaben. Besonders Unternehmen, welche sich schon immer in datenintensiven Industrien bewegt haben, sind vertraut mit dieser Phase. Die datenbasierte Kundenbetreuung von Finanzdienstleistern anhand von Customer-EquityModellen ist ein passendes Beispiel.

3. Marketing-Automatisierung: Sobald die Validität von analytischen Modellen in einem Unternehmen über einen langen Zeitraum bestätigt werden konnte, bietet sich die Möglichkeit zur Automatisierung derselben. Auf Basis von Algorithmen und komplexeren Datengrundlagen werden weitgehend eigenständige Prozesse der Kundeninteraktion durchgeführt. Beispielsweise die Personalisierung von Werbebotschaften oder das Ab-

\section{Abb. 1 Entwicklungsschritte in der Marketingautomatisierung}

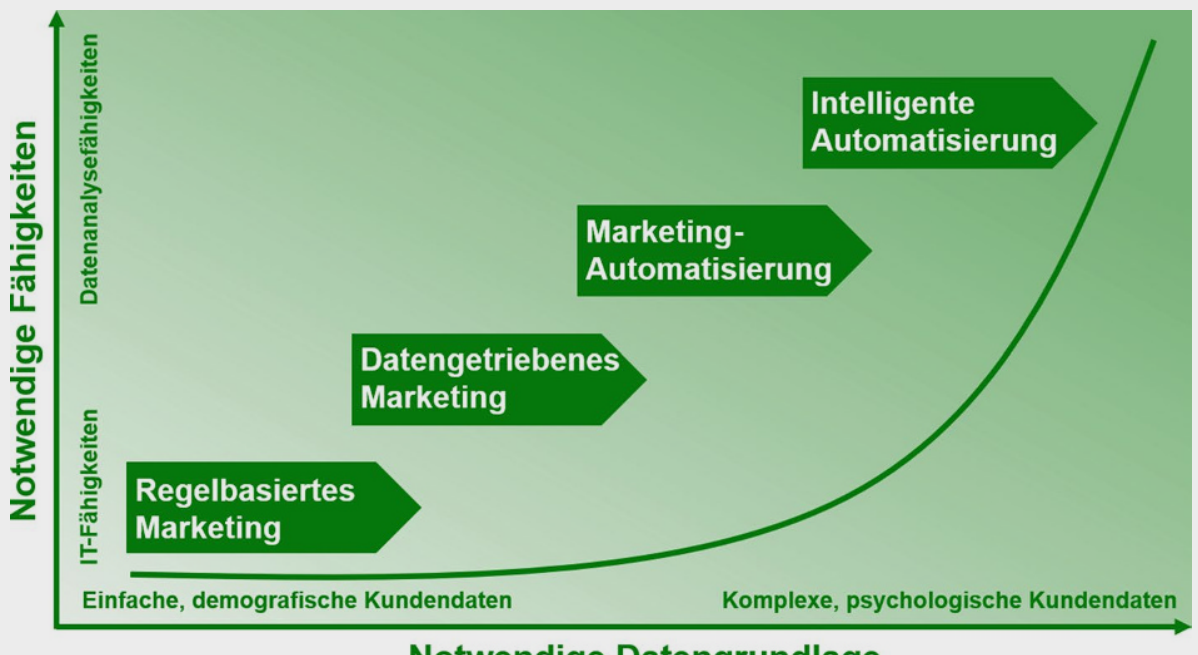

Notwendige Datengrundlage 
fangen von einfachen Kundenanfragen durch Chatbots. Diese Prozesse können bereits lernfähig sein, sind aber in ihrer Anwendung stark beschränkt auf wenige und spezifisch definierte Aufgaben.

4. Intelligente Automatisierung: Die letzte Phase bringt Unternehmen näher an die intelligente Automatisierung, also die Entwicklungsstufe des selbstständigen maschinellen Lernens und Entscheidens. Ob diese Phase der Automatisierungswelle bereits von jemandem erreicht wurde, steht zur Debatte. Natürlich bewerben Silicon-Valley-Tech-Giganten wie Amazon und Google oder asiatische Super-Apps wie WeChat oder Grab gerne ihre voll automatisierten Services, doch für die Mehrheit der Marketingprozesse bleibt die intelligente Automatisierung Zukunftsmusik.

Diese vier Phasen sind nicht nur rein deskriptiv zu verstehen, sondern als notwendige Schritte auf dem Weg zur intelligenten Automatisierung. Die vergleichsweise „simpleren“ Phasen in diesem Prozess gehen einher mit der Akquise von spezifischen Unternehmensressourcen - beispielsweise dem Aufbau einer soliden Kundendatenbank, dem Ausbau der digitalen Kompetenzen des Marketingteams oder der Akquise eines unternehmensweiten Datensilos. Diese Unternehmensressourcen führen optimalerweise zu höherer Rendite, welche wiederum in das Entwickeln weiterer Automatisierungskompetenzen investiert werden kann.

\section{Respekt als Fundament des Kundendialogs}

Die Automatisierung von Prozessen wird oft als Ziel in sich selbst dargestellt. Automatisierung bedeutet Effizienz und gesparte Zeit, welche wiederum anderswo produktiv eingesetzt werden kann. Dabei wird oft außer Acht gelassen, dass die grundlegenden Regeln von erfolgreichen Kundengesprächen auch in automatisierten Prozessen von Bedeutung sind. Studien zeigen, dass Kunden hyperpersonalisierte Werbenachrichten [7] oder lange Interaktionen mit Chatbots $[8,9]$ oft als störend empfinden. Dies liegt daran, dass im Design vieler Marketingapplikationen die Regeln des Dialogmarketings oft verletzt werden. So geht es nicht darum, den Kunden automatisiert mit möglichst vielen (unrelevanten) Inhalten zu überfluten, wie es mit gezielten Werbekampagnen auf sozialen Medien oft der Fall ist. Stattdessen kann eine Ausrichtung auf die etablierten Regeln des Dialogmarketings sicherstellen, dass sich Kunden auch in automatisierten Prozessen respektiert fühlen.

Im Mittelpunkt des Dialogmarketings steht eine einfache Formel: „Das große ,JA', die Zustimmung des Kunden zu einem Angebot, tritt nur dann ein, wenn während des schriftlichen Dialoges die Summe vieler vorausgehenden kleiner ,Jas‘ stets größer bleibt als die Summe der kleinen ,Neins‘. Ganz gleich, über welches Medium der Dialog geführt wird“ [10]. Diese Ausrichtung auf die „einfachen“, aber trotzdem wichtigen Zusagen von bestehenden Leads können, ohne die eigenen Kunden zu verärgern, automatisiert werden. Marketing-Guru Seth Godin [11] erkannte dies ebenfalls schon früh und formulierte drei Regeln für Kundeninteraktionen, welche leicht aktualisiert im Kontext der Automatisierung nur an Relevanz gewonnen haben:

\section{Zusammenfassung}

- Marketingautomatisierung benötigt eine schrittweise Skalierung von unternehmensinternen Kompetenzen und Datengrundlagen.

- Die Berücksichtigung der Prinzipien des Dialogmarketings ist zentral für das Design von kundenzentrierten, automatisierten Prozessen.

- Der frühzeitige Aufbau von Soft Skills wird die Integration von Mensch und Maschine in automatisierten Prozessen erleichtern. 


\section{Kernthese 1}

\section{Intelligente Automatisierung} ist kein Projekt, das man abschließt, sondern ein Prozess, den man anstößt.
- Vorhersehbarkeit: Automatisierung sollte nur in Kontexten eingesetzt werden, wo der Kunde dies auch vorhersehen und erwarten kann. So sollten Produktberatungen oder Beschwerden irgendwann zu einem Gespräch mit einer Person und nicht mit einem Chatbot führen.

- Personalisiert: Automatisierung beruht, zumindest unter der Datenschutz-Grundverordnung der Europäischen Union (DSGVO), auf freiwillig geteilten Kundendaten. Dementsprechend erwarten Kunden einen Mehrwert für das Teilen ihrer Daten, den sie sonst nicht erhalten hätten [12]. Automatisierte Nachrichten und Marketinginhalte sollten dementsprechend auf den spezifischen Kunden zugeschnitten sein.

- Relevant: Die vermutlich wichtigste Regel der drei stellt die Frage: „Wurde dieser Prozess automatisiert, um dem Kunden zu helfen, oder um das Unternehmen zu entlasten?" Haben wir einen Chatbot, damit Kunden schneller an die für sie relevanten Inhalte kommen, oder weil unsere Kundenbetreuung sonst überlastet wäre? Schicken wir personalisierte Newsletter, weil unser Algorithmus relevante Empfehlungen erstellen kann, oder weil wir sonst keine Inhalte zu teilen hätten?

All diese Regeln sollten einhergehen mit einem allgemeinen Respekt für die Zeit der eigenen Kunden. Während allen automatisierten Prozessen sollten Kunden die Chance haben, die Inhalte, die Datenerhebung und die Interaktionsfrequenz mit dem Unternehmen komplett bearbeiten zu können.

\section{Kundenprozesse als Orientierungsrahmen für die Auto- matisierung}

Die zahlreichen Automatisierungsmöglichkeiten, die Marketer zur Verfügung stehen, könnten bereits heute große Teile der Customer Journey abdecken. Jedoch genau wie sich einzelne automatisierte Prozesse an die Regeln des Dialogmarketings halten sollten, um effektiv zu sein, sollten automatisierte Prozesse mit Bedacht in die Customer Journey (siehe Abb. 2) integriert werden. Welche Technologien wo in die Customer Journey passen, hängt von ihrem grundlegenden Zweck ab:

- Bedürfnisse entdecken, Lösungssuche und Alternativen abwägen: gefragt sind Reichweite und Relevanz. Auch wenn von den meisten Plattformen die Push-Fähigkeit ihrer Werbeanzeigen angepriesen wird, liegt das vermutlich größte Potenzial der Marketingautomatisierung in dieser Phase in der Generation einer Pull-Wirkung. In Kombination mit einem gut kuratierten Content Marketing, können sowohl aktive als auch passive Automatisierungstechniken verwendet werden, um potenzielle Käufer besser zu erfassen. Aktive Ansätze nutzen Chatbots oder geleitete Fragen, um Kunden zu den richtigen Landingpages zu leiten, beispielsweise durch einfache Anleitungen wie: „Möchten Sie mehr über dieses Thema erfahren?" Zusammen mit passiven Ansätzen, zum Beispiel dem Verfolgen von Kundenpräferenzen durch Cookies, können aktuelle Programme diese aktiven Nachrichten zusätzlich personalisieren und somit erfahren, in welcher Phase der Customer Journey sich ein potenzieller Kunde befindet [14]. 
- Entscheidungen treffen und den Kauf abschließen: der Fokus liegt auf den Conversions. Die vermutlich wichtigste Phase in der Customer Journey sollte je nach Geschäftsmodell mit weniger Automatisierung auskommen - schließlich bleibt für die Abfrage und Erfüllung von individuellen Kundenbedürfnissen der direkte Dialog die Königsdisziplin. Trotzdem haben sich in den letzten Jahren ein paar Techniken bewährt, um den Kunden zum Kauf zu begleiten. Eine schnellere Antwortzeit durch ein intelligentes Ticketing-System oder die Beantwortung von Anfragen über sämtliche Channels hinweg sind nur zwei Beispiele. Datenintensivere Systeme, wie dynamische Preissetzung oder die Personalisierung von Touchpoints (beispielsweise mit personalisierten Angeboten im Onlinestore), werden in Zukunft auch für nicht digitale Geschäfte eine immer wichtigere Rolle spielen.

- Nutzungsphase und Beziehung aufbauen: Treue ist die wichtigste Messgröße. Die Automatisierung der letzten Phase der Customer Journey hängt stark mit dem jeweiligen Unternehmen zusammen. Einfache Automatisierungsprozesse wie personalisierte Newsletter und Angebote tragen dazu bei, den Kunden nicht aus den Augen zu verlieren. Etwas komplexere Prozesse, wie vorausahnende Wartungen („predictive customer service“), beruhen auf jahrelanger Erfahrung mit den eigenen Produkten und kreieren die Kauferfahrung, welche aus normalen Kunden Markenbotschafter macht.

Viele Automatisierungsprojekte scheitern, weil die Zielsetzung der eingesetzten Applikationen nicht zu der Phase in der Customer Journey passt,

\section{Kernthese 2}

\section{Kundenprozesse bilden den Orientierungsrahmen für effektive Automatisierung.}

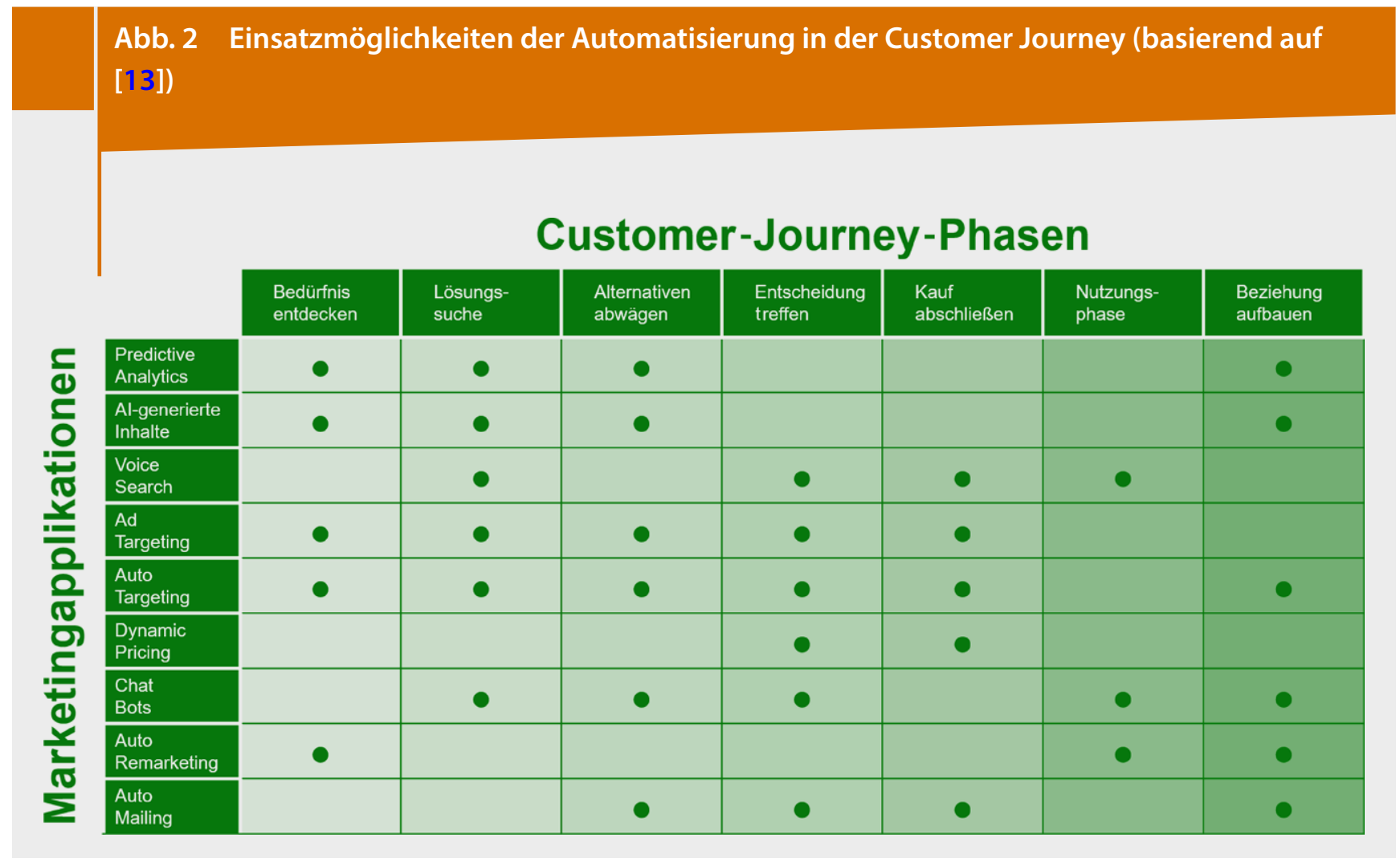


in der man sie eingesetzt hat. Ein Beispiel wäre die Messung von Likes und Kommentaren („vanity metrics“) bei Marketinginhalten, welche eigentlich für die Erhöhung der Verkaufszahlen gedacht waren. Dementsprechend ist es wertvoll, sich stets zuerst an den Kundenprozessen zu orientieren.

\section{Lernen, mit der Maschine zu kooperieren}

Trotz ihres hohen Innovationspotenzials stellt Automatisierung auch eine Gefahr für die von Menschen belegten Stellen dar. Diese Veränderung konnte bereits in den meisten Industriesektoren beobachtet werden, deren automatisierte Jobs praktisch komplett durch den Dienstleistungssektor aufgefangen werden mussten. Eine ähnliche Verschiebung wird es auch im Dienstleistungssektor geben - aber dieses Mal werden graduell bestimmte Aufgabentypen anstatt ganze Jobs abgelöst werden (siehe Abb. 3; [1]). Unternehmen, welche sich auf der Reise zur intelligenten Automatisierung bewegen, sollten deshalb zwei Fragen stellen: Welche Kompetenzen sollten im Humanressourcen(HR)-Pool gefördert werden und wie wird die Integration von automatisierten Prozessen und menschlichen Inputs aussehen?

Es mag wenig überraschend sein, dass Zukunftsforscher schon lange davon ausgehen, dass repetitive und leichte mechanische Aufgaben noch zu unseren Lebzeiten größtenteils automatisiert werden könnten. Vermutlich überraschender ist, dass die momentan in vielen Universitäten angepriesenen analytischen Fähigkeiten (zum Beispiel Datenauswertung, Statistik etc.) nicht mehr sicher vor der Automatisierung sind. Unternehmen sollten dementsprechend ihre Marketingabteilung früh umschulen auf die empathischen und intuitiven Soft Skills, welche für die eigenständige Weiterentwicklung von Projekten und die kreative Anwendung von automatisierten Werkzeugen notwendig sein werden, um einen kompetitiven Vorteil zu erlangen [1].

\section{Abb. 3 Phasenmodell der Automatisierung von Marketingaufgaben (basierend auf [1])}

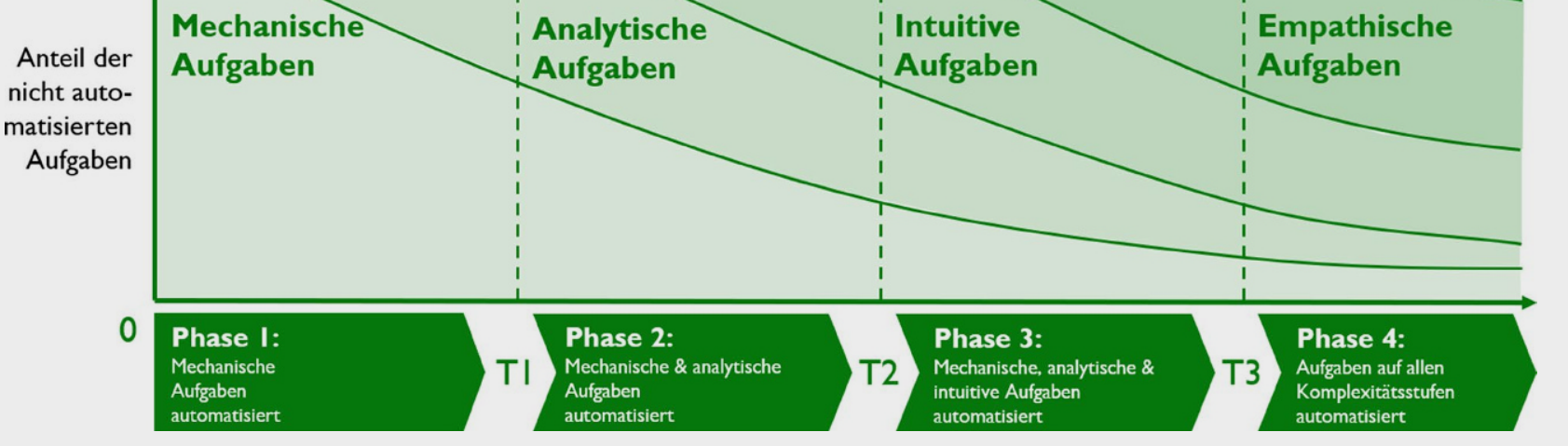


Es sind auch diese Soft Skills, welche in der Integration von automatisierten Prozessen mit menschlichen Agenten zentral sind. Letztere sind und bleiben die notwendigen Schnittstellen zwischen den in automatisierten Prozessen gesammelten Informationen und den menschlichen Agenten, welche auf der Basis dieser Informationen eine Leistung erbringen müssen. Dabei sind eine schnelle Auffassungsgabe und viel Empathie notwendig, um komplexe Daten den Bedürfnissen des Empfängers angepasst zu vermitteln [8].

\section{Die Gefahren auf dem Weg beachten}

Ob die von Gartner prognostizierte Automatisierungswelle [2] schon bald eine Realität sein wird, oder nicht, wird sich zeigen. Die negativen Konsequenzen einer extensiven Marketingautomatisierung sind hingegen bereits jetzt absehbar. Konsumenten fühlen sich bereits heute online oft in ihrer Privatsphäre verletzt und nicht in der Lage, sich gegen solche Verletzungen zu verteidigen [15]. Die Technologien hinter der Marketingautomatisierung (Stimmerkennung, das Internet of Things (IoT), „predictive analytics“ etc.) werden die Verwundbarkeit von Konsumenten in Bezug auf Datenschutz und Privatsphäre drastisch verschärfen [16] und die meisten Unternehmen verfügen nicht über die Ressourcen, um die Daten ihrer Kunden adäquat zu schützen. Seit dem Inkrafttreten der DSGVO in 2018 sind dem europäischen Datenschutzverantwortlichen über 270.000 Verletzungen der Datensicherheit gemeldet worden. Die Mehrheit dieser Verletzungen kann auf fehlende Kompetenzen vonseiten der Unternehmen zurückgeführt werden: inakkurates Data-Mapping, intransparente Erhebungsverfahren, ungenügende Sicherheit in der Aufbewahrung oder dem Sammeln von zu viel oder zweckfremden Daten. Es entsteht ein Spannungsverhältnis zwischen den Datenbedürfnissen der Marketingautomatisierung und den sich verschärfenden Datenschutzmaßnahmen in der EU und den USA [17].

Diese Hindernisse sind nicht unumgänglich - eine Untersuchung von 32 Fallstudien zur Implementierung der DSGVO-Richtlinien hat gezeigt, dass eine frühe Ausrichtung der Datenerhebungsprozesse auf die gesetzlichen Richtlinien in Kombination mit einer parallelen Skalierung der notwendigen menschlichen Ressourcen zum Erfolg führt. In Kombination mit einer frühen Adaption von Anonymisierungstechnologien besteht die Möglichkeit, von Marketingautomatisierung zu profitieren und sich gleichzeitig mit höheren Datenschutzstandards von der Konkurrenz abzuheben.

\section{Fazit - der Weg ist das Ziel}

Der Aufbau der notwendigen Ressourcen zur intelligenten Automatisierung wird für die meisten Unternehmen ein evolutionärer Prozess mit einigen intensiven Lernschleifen sein. Deshalb gilt es, die folgenden Ratschläge für unterwegs zu beachten:

1. Einzelne Schritte: Die Reise hin zur intelligenten Automatisierung führt durch vier grobe Phasen: (1) regelbasiertes Marketing, (2) datengetriebenes Marketing, (3) Marketingautomatisierung und schließlich (4) intelligente Automatisierung. Der erste Schritt ist es, herauszufinden, in

\section{Kernthese 3}

Die Gefahren der Automatisierung zeichnen sich schon heute ab und müssen frühzeitig adressiert werden. 


\section{Handlungsempfehlung}

- Prüfen, in welcher Entwicklungsphase der Automatisierung sich das eigene Unternehmen befindet

- Automatisierungsprozesse in den größeren Kontext der Customer Journey stellen

- Frühzeitig in unternehmensinterne Fähigkeiten und Ressourcen investieren

welcher Phase sich das eigene Unternehmen befindet - denn für eine erfolgreiche Implementierung sollte keine der vier Phasen übersprungen werden.

2. Respekt für den Kunden: Automatisierte Prozesse in jeder Phase der Reise dürfen grundlegende Marketingerkenntnisse nicht aus den Augen verlieren. Gemäß den Prinzipien des Dialogmarketings sollte stets darauf geachtet werden, dass mit dem Kunden nur mit seiner Zustimmung (1) vorhersehbare, (2) personalisierte und (3) relevante Dialoge geführt werden.

3. Integration in die Customer Journey: Selbst wenn die gesamte Customer Journey automatisiert werden könnte, ist dies vermutlich keine gute Idee. Stattdessen sollte das Ziel sein, die Interaktionen zwischen Touchpoints mit tiefem Kundenengagement (beispielsweise das Suchen nach Produkten) durch Automatisierung zu beschleunigen, damit mehr Fokus auf die menschlichen Schnittstellen und entscheidenden Momente gelegt werden kann.

4. Interaktionen zwischen Mensch und Maschine verstehen: Durch die Automatisierung werden interpersonelle Soft Skills und Kreativität nur an Wichtigkeit gewinnen. Es gilt deshalb, dass die entsprechenden Kompetenzen, Abläufe und Trainings schon früh aufgebaut werden, damit menschliches Potenzial in automatisierten Prozessen voll ausgeschöpft werden kann.

5. Die Gefahren früh adressieren: Die Gefahren der Marketingautomatisierung gehen weit über einzelne Unternehmen hinaus. Das steigende Bedürfnis nach Kundendaten erhöht die Verletzbarkeit der Privatsphäre von Konsumenten. Dementsprechend bietet sich die Chance, sich am Markt durch eine höhere Datenschutzkompetenz strategische Vorteile zu verschaffen.

Schließlich ist die Reise das Ziel. Unternehmen, welche die Reise bewusst antreten und jeden Schritt selbst gehen, werden sich des Potenzials dieser neuen Technologien bewusst werden und neue, überlegene Kundenerlebnisse schaffen. Andere, welche Schritte überspringen oder Gefahren missachten, werden sich irgendwann in einer Sackgasse wiederfinden.

Funding. Open access funding provided by University of St.Gallen.

Open Access. Dieser Artikel wird unter der Creative Commons Namensnennung 4.0 International Lizenz veröffentlicht, welche die Nutzung, Vervielfältigung, Bearbeitung, Verbreitung und Wiedergabe in jeglichem Medium und Format erlaubt, sofern Sie den/die ursprünglichen Autor(en) und die Quelle ordnungsgemäß nennen, einen Link zur Creative Commons Lizenz beifügen und angeben, ob Änderungen vorgenommen wurden.

Die in diesem Artikel enthaltenen Bilder und sonstiges Drittmaterial unterliegen ebenfalls der genannten Creative Commons Lizenz, sofern sich aus der Abbildungslegende nichts anderes ergibt. Sofern das betreffende Material nicht unter der genannten Creative Commons Lizenz steht und die be- 
treffende Handlung nicht nach gesetzlichen Vorschriften erlaubt ist, ist für die oben aufgeführten Weiterverwendungen des Materials die Einwilligung des jeweiligen Rechteinhabers einzuholen.

Weitere Details zur Lizenz entnehmen Sie bitte der Lizenzinformation auf http://creativecommons.org/licenses/by/4.0/deed.de.

\section{Literatur}

[1] Huang, M.-H., \& Rust, R. T. (2018). Artificial intelligence in service. Journal of Service Research, 21, 155-172.

[2] Panetta, K. (2020). Top strategic technology trends for 2021. Gartner Trends. Stamford: Gartner.

[3] Sutcliff, M., Narsalay, R., \& Sen, A. (2019). The two big reasons that digital transformations fail. https://hbr.org/2019/10/the-two-big-reasons-that-digitaltransformations-fail. Zugegriffen: 26. Juli 2021.

[4] Schögel, M. (2016). Marketing automation, machine learning und artificial intelligence. Marketing Review St. Gallen, 2016, 3.

[5] Bornet, P., Barkin, I., \& Wirtz, J. (2020). Intelligent automation - welcome to the world of hyperautomation

[6] Mero, J., Tarkiainen, A., \& Tobon, J. (2020). Effectual and causal reasoning in the adoption of marketing automation. Industrial Marketing Management, 86, 212-222.

[7] Kim, T., Barasz, K., \& John, L. K. (2019). Why am i seeing this ad? The effect of ad transparency on ad effectiveness. Journal of Consumer Research, 45, 906-932.

[8] Castillo, D., Canhoto, A. I., \& Said, E. (2020). The dark side of AI-powered service interactions: exploring the process of co-destruction from the customer perspective. Service Industries Journal. https://doi.org/10.1080/02642069.2020.1787 993.

[9] Hill, J., Randolph Ford, W., \& Farreras, I. G. (2015). Real conversations with artificial intelligence: a comparison between human-human online conversations and human-chatbot conversations. Computers in Human Behavior, 49, 245-250.

[10] Gerdes, J., \& Hesse, J. (2013). Dialogmarketing im Dialog. Wiesbaden: Gabler. https://doi.org/10.1007/978-3-658-02000-2.

[11] Godin, S. (1999). Permission marketing: turning strangers into friends and friends into customers. New York: Simon \& Schuster.

[12] Acquisti, A., Taylor, C., \& Wagman, L. (2016). The economics of privacy. Journal of Economic Literature, 54, 442-492.

[13] Chaffey, D. (2019). 15 applications of artificial intelligence in marketing

[14] Järvinen, J., \& Taiminen, H. (2016). Harnessing marketing automation for B2B content marketing. Industrial Marketing Management, 54, 164-175.

[15] Gotsch, M. (2020). Was hat das Marketing nach 2 Jahren DSGVO gelernt? Swiss Marketing Review, 2020(6), 4-9.

[16] Palmatier, R. W., \& Martin, K. D. (2019). The intelligent marketer's guide to data privacy. London: Palgrave Macmillan.

[17] DLA Piper (2020). DLA Piper GDPR data breach survey: January 2020 